\title{
Controlling dissociation by trapping trajectories in highly energetic states
}

\author{
A.K. de Almeida Jr. ${ }^{a}$, R. Egydio de Carvalho ${ }^{\text {a,* }}$, E.F. de Lima ${ }^{b}$ \\ ${ }^{a}$ Universidade Estadual Paulista-UNESP, Rio Claro-SP, Brazil \\ ${ }^{\mathrm{b}}$ Universidade Federal de São Carlos-UFSCAR, São Carlos-SP, Brazil
}

\section{H I G H L I G H T S}

- A particle is trapped in a state outside the potential well.

- The trap is a non-linear resonance island.

- Chaotic and point attractors prevent the particle photo-dissociation.

- Dissipation inhibits photo-dissociation for energies above the dissociation threshold.

- The model used is the perturbed Morse oscillator.

\section{A R T I C L E I N F O}

\section{Article history:}

Received 17 December 2015

Available online 4 January 2016

\section{Keywords:}

Morse potential

Non-linear resonance

Dissociation

Dissipation

Attractors

\begin{abstract}
A B S T R A C T
We consider the non-linear dynamics of a polar diatomic molecule under the action of laser-field interactions and in the presence of a dissipation mechanism, described by the classical damped and driven one-dimensional Morse oscillator. In the absence of laser fields and dissipation, the phase space consists of a negative-energy bound region and a positive-energy dissociative region. Laser-molecule interaction changes the phase space allowing transitions from the bound to the dissociative region through chaotic routes. We show that for a spatially dependent dipole force, resonances with positive energies allow the trapping of trajectories in pseudo-bound states. We also show that, upon the introduction of dissipation, there exist non-trivial point attractors as well as chaotic attractors, which capture the trajectories in pseudo-bound states. Consequently, in addition to the parameters associated with the laser-molecule interaction, the amplitude of the dissipation acts as a control parameter of the photo-dissociation dynamics.
\end{abstract}

(C) 2015 Elsevier B.V. All rights reserved.

\section{Introduction}

Forced and dissipative non-linear oscillators can model a variety of important phenomena. One example is the damped and driven Morse oscillator, which can model vibrational excitations, breaking of a chemical bond of a polyatomic molecule by laser fields, multiphoton excitations and dissociation of hetero-nuclear diatomic molecules in a gaseous cell under high pressure [1-3].

In the absence of interactions with time-dependent external fields as well as dissipation, the Morse oscillator phase space can be divided into two parts: the bound region with negative energies and the unbound, or dissociative region with positive energies. The interaction of the unperturbed oscillator with an external driving field alters the phase space permitting

\footnotetext{
* Corresponding author.

E-mail addresses: fenixcinza@yahoo.com.br (A.K. de Almeida Jr.), regydio@rc.unesp.br (R. Egydio de Carvalho), eflima@ufscar.br (E.F. de Lima).
} 
transitions from the bound to the unbound region through chaotic trajectories [4-8]. This photo-dissociation process can be regarded as a half collision, which is a particular case of chaotic scattering [9-15]. Although the majority of contributions investigating the Morse oscillator have been carried out for non-dissipative cases, including classical, semi-classical and quantum approaches [4-8,16-18], there are nevertheless, a number of works with the dissipative Morse oscillator. For instance, in Ref. [19] the bi-stable and chaotic behavior of the system were studied, while in Ref. [20] bifurcations and fixed-point diagrams for two damping constants were investigated, and in Ref. [21] the analytic structure of the Morse oscillator was considered. A detailed study of this system considering the variation of five parameters was performed in Ref. [22]. More recently, a study on the control of the chaotic dynamics through slaver-master feedback scheme has been presented in Ref. [23]; and in Ref. [24] investigations about vibrational resonances for the damped Morse oscillator driven by bi-harmonic forces were carried out. A common feature in all these works is that the periodic forcing of the oscillator is independent of the position.

The interaction energy of a polar diatomic molecule under the action of a laser-field depends on the permanent dipole function of the molecule as well as on the laser time-dependent electric field. A linear dipole function, which may be valid for small oscillation amplitudes, implies a force that is independent of the relative distance of the atomic pair. However, realistic molecular permanent dipoles generally have non-linear spatial behavior, leading to a force acting on the molecule which does depend on the inter-nuclear distance [25-27]. There is, in fact, an increasing interest in studying the role of the dipole function in controlling the dynamics of molecular systems $[28,29]$. We have investigated in previous works how this spatial dependence of the dipole influences the dissociation dynamics [30-32]. We have shown that the spatial variation of the dipole function through controllable parameters has a considerable impact in the system dynamics, inhibiting dissociation for some values of these parameters.

In the present work, we consider the classical damped Morse oscillator driven by a space-dependent and time-periodic force. In particular, we investigate the role played by a parameter related to the spatial shape of dipole function and by a parameter that dictates the dissipation strength. The paper is organized as follows. In Section 2, we describe the model system. In Section 3, we present and discuss the results of the calculations. Finally the conclusions are drawn in Section 4.

\section{The Morse system, the laser field perturbation and dissipation}

We consider the dynamics of polar diatomic molecules interacting with a laser-field, described by the classical driven one-dimensional Morse oscillator. In considering this model, we are neglecting both rotational and electronic excitations of the molecule. Although this approximation might be fulfilled by appropriate experimental apparatus, for instance by applying short linearly-polarized laser pulses in the infrared domain, the main reason for considering a non-perturbed Hamiltonian with one degree of freedom is that it captures the essence of the Physics involved in the breaking of the chemical bond, allowing us to focus on the relative distance of the nuclei.

For the non-dissipative case, the total Hamiltonian describing the system can be written in two parts. The first one is the Hamiltonian $H_{0}(x, p)$ which describes the unperturbed relative motion of the nuclei [5-8].

$$
H_{0}(x, p)=\frac{p^{2}}{2}+\frac{1}{2}\left(\mathrm{e}^{-2 x}-2 \mathrm{e}^{-x}\right),
$$

where $x$ stands for the inter-nuclear distance and $p$ for the molecule momentum. If the molecule energy is negative, it is in the bound energy region, while for positive energies, it is in the unbound region. Both regions are separated by the limiting energy, $E=0$, which corresponds to a separatrix curve in the phase space. Therefore, the bound energies correspond to libration tori and the unbound energies correspond to rotation tori in the phase space of the system governed by Eq. (1).

The second term, $H_{1}(x, t)$, is the perturbation accounting for the laser-molecule interaction,

$$
H_{1}(x, t)=-\varepsilon(t) \mu(x, \eta),
$$

where $\varepsilon(t)$ is the time-dependent external laser electric field and $\mu(x, \eta)$ is the non-linear dipole function of the molecule. The external laser field represents a monochromatic pulse, acting during an adjustable time interval $T$, given by

$$
\varepsilon(t)=\varepsilon_{0} \sin (\omega t),
$$

with $\varepsilon_{0}$ and $\omega$ being the field amplitude and its frequency, respectively. The dipole function was taken from previous work [30],

$$
\mu(x, \eta)=\frac{\sin \left[\eta\left(x+x_{e}\right)\right] \mathrm{e}^{-\xi\left(x+x_{e}\right)^{4}}}{\eta},
$$

where $\eta$ and $\xi$ are dimensionless adjustable parameters, $x_{e}$ is related with the minimum of the Morse potential but is also adjustable. $\eta$ plays the role of a deformation parameter introducing oscillations on the dipole function as it increases [30]. As $\eta$ decreases, it is possible to model other known dipole functions, as for instance the one of the HF molecule. For small enough values of $\eta, \mu(x, \eta)$ does not oscillate in space and the mechanism of photo-dissociation can be explained through the splitting of the separatrix. Large values of $\eta$ imply in oscillatory forms for $\mu(x, \eta)$, which can lead to a regularization of the phase space and consequently to the inhibition of dissociation. 
The representative Hamiltonian for the purpose of this paper is described by the following one-dimensional and dimensionless Hamiltonian [30],

$$
H(x, p, t)=\frac{p^{2}}{2}+\frac{1}{2}\left\{\mathrm{e}^{-2 x}-2 \mathrm{e}^{-x}\right\}-\varepsilon_{0} \sin (\omega t) \frac{\sin \left[\eta\left(x+x_{e}\right)\right] \mathrm{e}^{-\xi\left(x+x_{e}\right)^{4}}}{\eta} .
$$

In order to introduce dissipation in the problem, we need to obtain the Newtonian formulation for the system, which can be done from the Hamiltonian function given in Eq. (5), resulting in,

$$
\ddot{x}=\left[\mathrm{e}^{-2 x}-\mathrm{e}^{-x}\right]+\left[\frac{\varepsilon_{0} \sin (\omega t) \mathrm{e}^{-\xi\left(x+x_{e}\right)^{4}}}{\eta}\right] \cdot\left\{\eta \cos \left[\eta\left(x+x_{e}\right)\right]-4 \xi\left(x+x_{e}\right)^{3} \sin \left[\eta\left(x+x_{e}\right)\right]\right\},
$$

which can be easily converted in the two differential equations $\dot{x}=p$ and $\dot{p}=\ddot{x}$.

We introduce a dissipative term linearly proportional to the molecule velocity, $-\gamma \dot{x}$ where $\gamma$ is the dissipation coefficient. Therefore, Newton's equation becomes $\ddot{x}=f(x, t)-\gamma \dot{x}=f(x, t)-\gamma p$, in such way that the dissipative term is explicitly introduced in Eq. (6). In order to carry out the numeric study we have to fix some parameters, since there are 6 free parameters: $\varepsilon_{0}, \omega, \eta, \xi, x_{e}$ and $\gamma$. Attaching all units to the problem, there are 10 free parameters. However, some of these parameters appear in pairs in the definition of the dimensionless variables. This fact gives us freedom to fix some of these parameters. Details of the transformations to dimensionless units can be found in Ref. [30].

In order to investigate the molecular photo-dissociation, we define the classical dissociation probability as the ratio between the number of particles that reaches the positive-energy region in phase space with large inter-nuclear distance $(x>5)$ by the total number of particles initially placed in the short-range of the potential well,

$$
P=P(E>0, x>5)=\frac{N(E>0, x>5)}{N_{\text {Total }}} .
$$

The region of initial conditions in phase space is given by the rectangle of Fig. 1. Even though the limiting value $x=5$ is somewhat arbitrary, the force exerted by the external laser field for $x=5$ is negligible. Thus, although the definition of photo-dissociation, Eq. (7), is not the usual one, it accounts for the number of trajectories that escape from the influence of the potential, capturing the essence of the photo-dissociation process.

\section{Results}

As usual, we define a Poincare section as the plane corresponding to the state of the system at each period of the driving force. For all numerical calculations, we consider the energy obtained through the following procedure: the equations of motion for the perturbed system are integrated and when a trajectory cross the Poincaré section, the values of $x$ and $p$ are collected and substituted into the unperturbed Hamiltonian $H_{0}$. We have evaluated the dissociation probability for $\gamma=0$ and for $\gamma \neq 0$. Excepted when explicitly stated otherwise, we have used the following set of parameters,

$$
\varepsilon_{0}=2.5, \quad \omega=\sqrt{3}, \quad \eta=1, \quad \xi=1, \quad x_{e}=1 .
$$

Concerning the numerical methods, we have used for the integration of the equations of motion the Runge-Kutta method of 4 th order (RK4) with fixed step, whose dimensionless time-step was 10-4. We have decreased systematically this timestep in order to adjust an optimal time-step and for all smaller time steps, we have obtained the same results. Neither the point attractors nor the chaotic attractors have changed in any fashion.

Fig. 1 shows the level curves of the Morse potential in the phase space $(x, p)$, i.e. constant energy curves of $H_{0}$. We observe rotation and libration tori for positive and negative energies respectively, and a quasi-separatrix for the energy -0.0068 . The rectangle $-0.9 \leq x \leq 1.1,-0.5 \leq p \leq 1.2$ is a region involving libration and rotation that was defined as the region of the initial conditions. The level curves in this region have energies in the range [-0.5, 0.5]. When the laser field is acting in the system, there is a special region, marked in blue and red colors inside the rectangle, for the initial conditions. This is a region with resonance islands with libration tori permeated by secondary resonance islands and chaotic trajectories. We call this region a resonant region.

Fig. 2 shows a magnification of the resonant region of Fig. 1, for which we particularly defined a grid with $(21 \times 21)$ initial conditions equally spaced in the rectangle $-0.9 \leq x \leq 0.1$ and $0.2 \leq p \leq 1.2$. A primary resonance of order $1: 3$, secondary broken resonances, invariant tori and a thin layer of chaos in black color can be observed. The structures in red have positive energies while the blue ones have negative energies. The chaotic sea in black color permeates both regions. This point addresses an interesting scenario: the molecules can have positive energy, while remaining trapped in a resonance island. Consequently, if a particle has an initial condition inside a resonance island with positive energy it remains trapped and does not dissociate. On the other hand, if the initial condition is out of the island the particle can dissociate.

Fig. 3 shows the photo-dissociation probability for $\gamma=0$ as a function of the deformation parameter $\eta$ for three final propagation times. We have set a grid of $(35 \times 35)$ initial conditions in the rectangle of Fig. 1. It can be observed a roughness in the curve for the shorter time, which can be explained as the effect of stickiness around the resonant islands, i.e. some particles are trapped in the resonance region. Concerning the initial conditions that start with negative energies, most of 


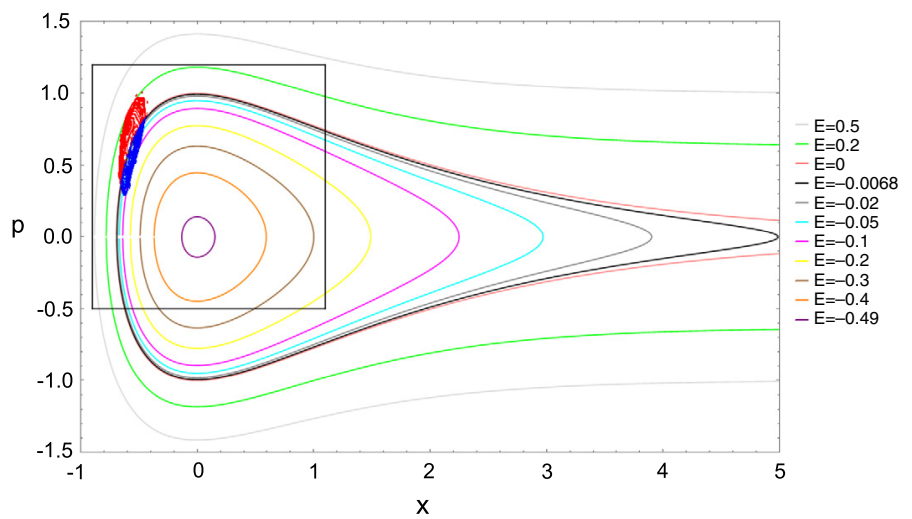

Fig. 1. The colored curves show different constant values for $H_{0}$ (the energies $E$ ). The rectangle is the region chosen for initial conditions. The red and blue "smudges" correspond to a resonance structure in the corresponding stroboscopic map for $\gamma=0$. (For interpretation of the references to color in this figure legend, the reader is referred to the web version of this article.)

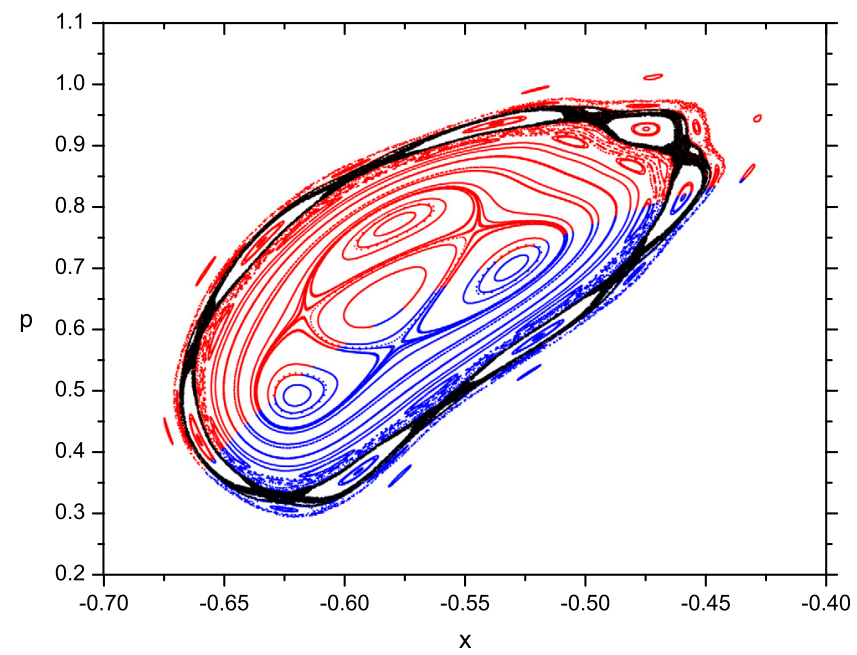

Fig. 2. Stroboscopic map showing the resonant region for $\gamma=0$ with a chaotic layer in black, resonance structures with positive energies in red and with negative energies in blue. (For interpretation of the references to color in this figure legend, the reader is referred to the web version of this article.)

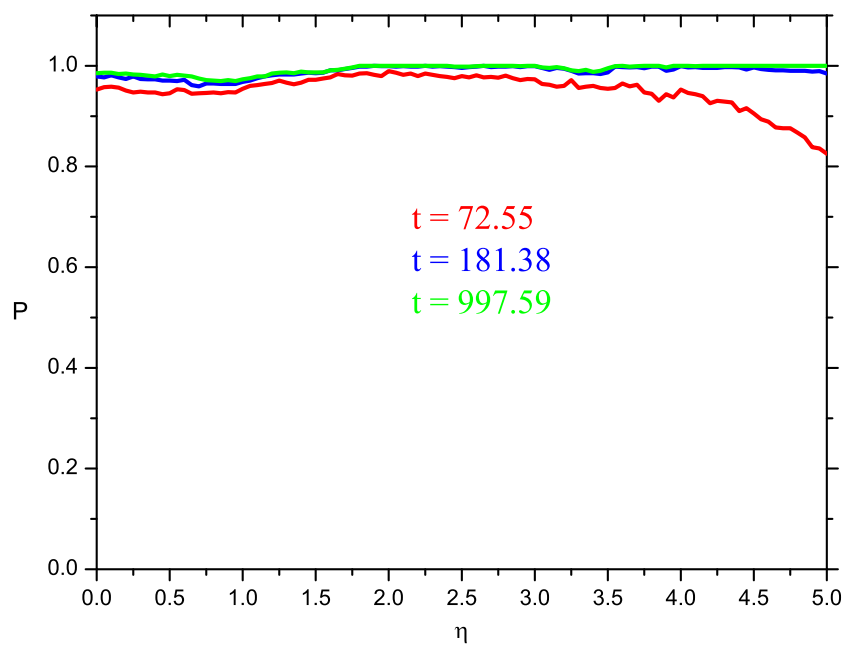

Fig. 3. Probability of photo-dissociation with $\gamma=0$, for three instant of time, as function of the dipole shape parameter $\eta$. 


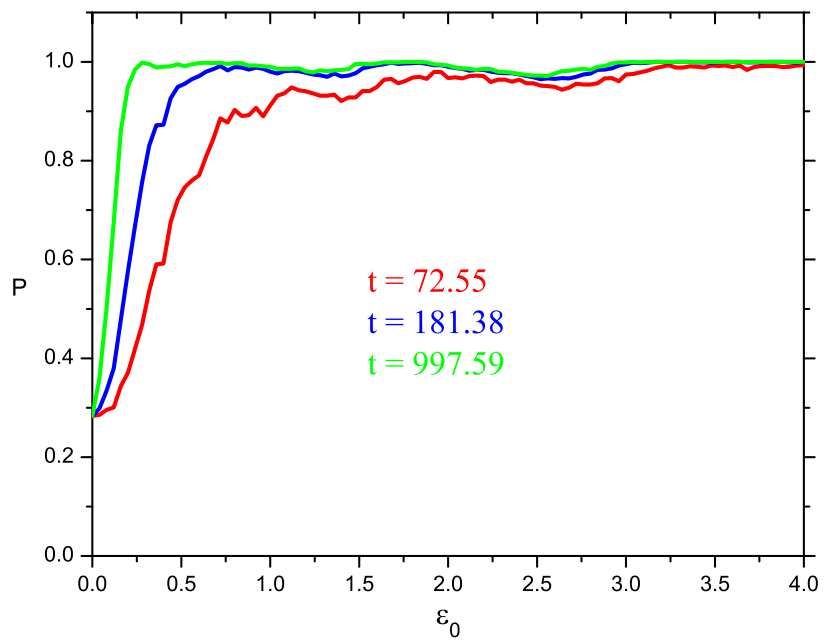

Fig. 4. Probability of photo-dissociation with $\gamma=0$, for three instant of time, as function of the amplitude parameter $\varepsilon_{0}$.

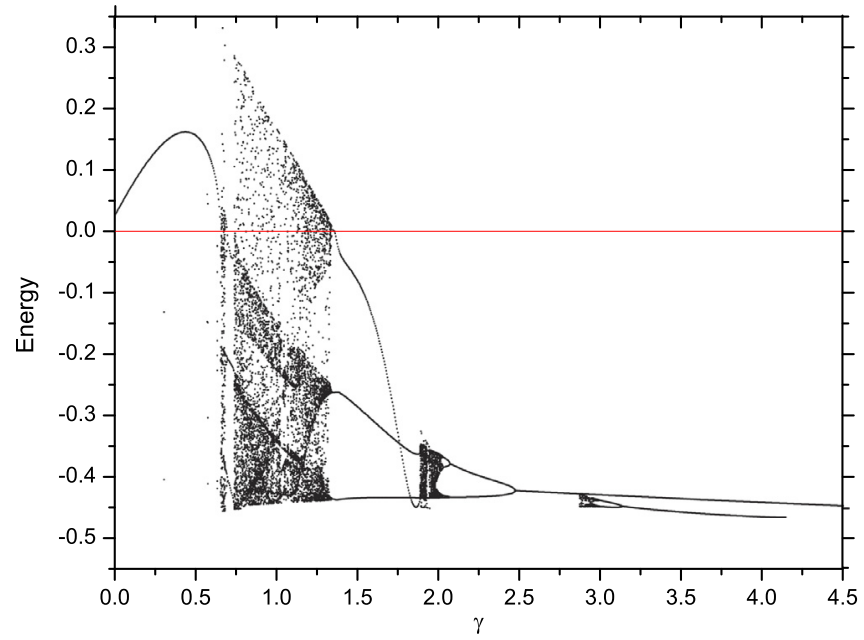

Fig. 5. Diagram of bifurcation for the energy as function of the dissipation parameter $\gamma$, with $\eta=1$ and $\varepsilon_{0}=2.5$. The red line is to guide the eyes for the energy of the separatrix. (For interpretation of the references to color in this figure legend, the reader is referred to the web version of this article.)

them are driven to positive energies since the amplitude $\varepsilon_{0}$ of the laser is considerably high. This point has been already reported in Ref. [30].

Fig. 4 shows the dissociation probability as a function of the field amplitude $\varepsilon_{0}$ for $\gamma=0$ and for the previously considered propagation times. It can be noted that the probability $P$ increases rapidly to the maximum value, in agreement with previous analysis.

In the following figures, we have set 28 initial conditions uniformly distributed in the rectangle of Fig. 1 . We have neglected the transient times so that all the trajectories are captured by the attractors.

Fig. 5 presents the molecule energy as a function of the dissipation parameter. For small values of $\gamma,<0.3$, the transient time is relatively large, which means that the trapping of the trajectories by the point attractor takes long propagation times. For $\gamma$ in the range $[\sim 0.5, \sim 2.0]$ a complex dynamics with chaotic and point attractors is observed, while for large values, $\gamma \sim 4$, it can be noticed that the point attractor corresponds to the neighborhood of the minimum energy of the potential.

In Fig. 6, the behavior of the inter-nuclear distance is shown as function of the dissipation parameter $\gamma$. The biggest chaotic attractor appears for $x$ in the range $[\sim 0.75, \sim 1.3]$. In the lower panel of Fig. 6 , we show the corresponding Lyapunov exponent, which is positive for the chaotic dynamics and negative for regular dynamics, while it is null at the bifurcation points. We have used the same initial condition $x=-0.4$ and $p=0.7$ for all calculations of the Lyapunov exponents. The fact that we have used only a unique initial condition for the calculation of the Lyapunov exponent prevents us to characterize eventual regions of multi-stability, since the orbit will fall in a basin of attractor of a specific attractor. We have applied Wolf's algorithm to calculate the Lyapunov exponents [33].

We define the transient time as the time the particle takes to leave the potential well, to attain large values of $x$ and subsequently to come back to the interaction region. Clearly, the transient time is related to the magnitude of the dissipation. 

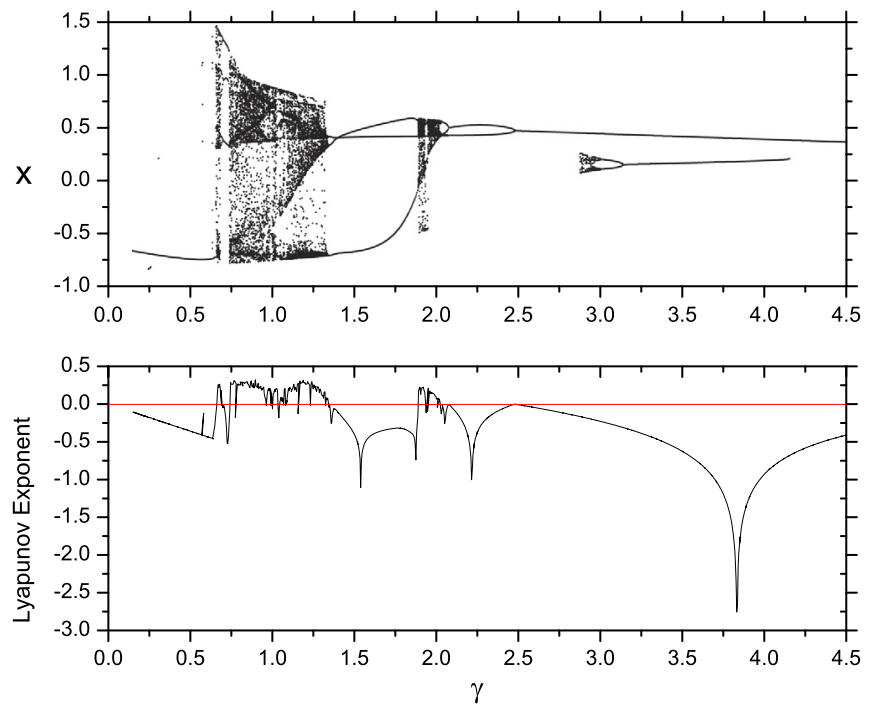

Fig. 6. Upper panel: diagram of bifurcation for the variation of the inter-nuclear distance, $x$, as function of the dissipation parameter $\gamma$, with $\eta=1$ and $\varepsilon_{0}=2.5$. Lower panel: the corresponding Lyapunov exponent. The red line is to guide the eyes. (For interpretation of the references to color in this figure legend, the reader is referred to the web version of this article.)

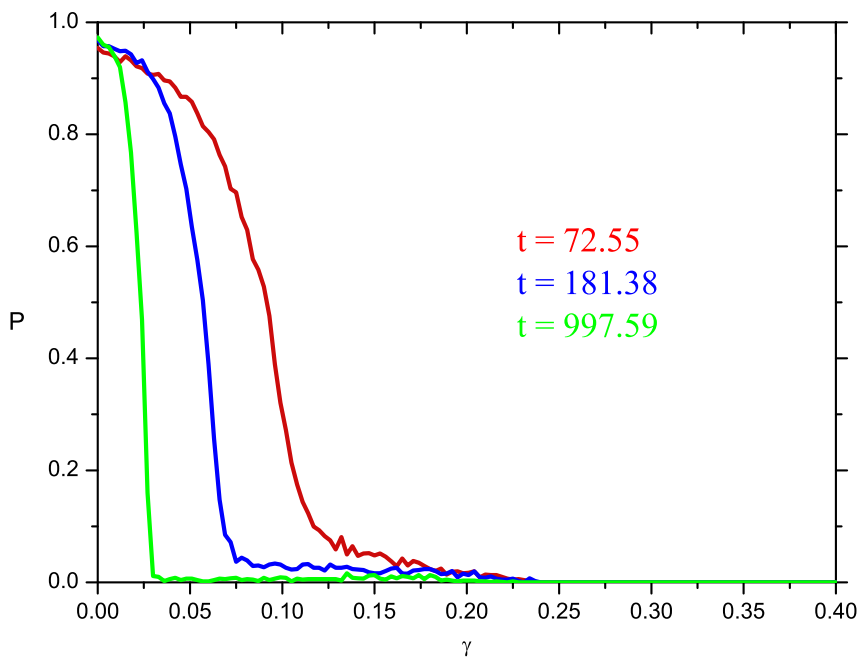

Fig. 7. Probability of photo-dissociation as function of the dissipation parameter $\gamma$ for three different final times.

In order to stress this relation, Fig. 7 shows the dissociation probability as a function of the dissipation parameter for three different propagation times. As $\gamma$ is increased, the dissociation probability tends to zero for all propagation times. However, for low values of the dissipation parameter, the transient time is longer. Thus, in order to avoid time-consuming calculations and to drive the trajectories to the attractors, we might use high values for the dissipation parameter.

In order to show how the introduction of dissipation may change the dissociation dynamics in a more realistic scenario we have set, as an example, the parameters of the HF molecule: $\xi=0.0029 ; \eta=0 ; x_{e}=2.135$ [17]. The unperturbed energy of the system is shown in Fig. 8 as a function of $\gamma$. As for the previous set of parameters, it is possible to trap the trajectories in highly energetic states by changing $\gamma$, though for the HF case, higher values of the dissipation parameter are needed.

Fig. 9 shows the energy as a function of $\eta$ for the parameters of Eq. (8). $\eta$ is varied from 0 to 5 and it can be noted a period-1 attractor up to $\eta \sim 1.5$ with positive energy, which means that even with positive energy the molecule will not dissociate. From $\eta \sim 1.5$ up $\eta \sim 3.0$ a chaotic attractor can be observed along with thin windows of regularity. From $\eta \sim 3.0$ up $\eta=5.0$, there are ranges with chaotic attractors and new windows of regularity.

Fig. 10 shows the inter-nuclear distance as function of the deformation parameter $\eta$ and should be compared with Fig. 9. The molecule remains localized at $x \sim-0.7$, for $\eta$ up to $\sim 1.5$, where there is a point attractor. From this value up to $\eta \sim 3.0$ the distance between the nuclei oscillates considerably, but the molecule does not dissociate. From $\eta \sim 3.0$ up to $\eta=5.0$ 


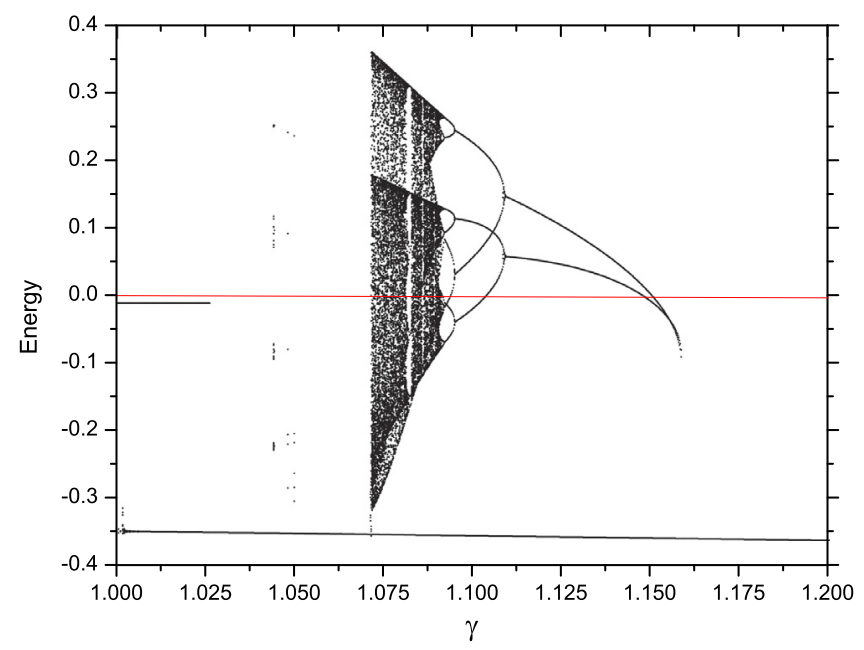

Fig. 8. Diagram of bifurcation for the energy as function of $\gamma$ for the parameters of the HF molecule: $\xi=0.0029, \eta=0, x e=2.135, \varepsilon_{0}=2.5, \omega=\sqrt{3}$. The red line is to guide the eyes. (For interpretation of the references to color in this figure legend, the reader is referred to the web version of this article.)

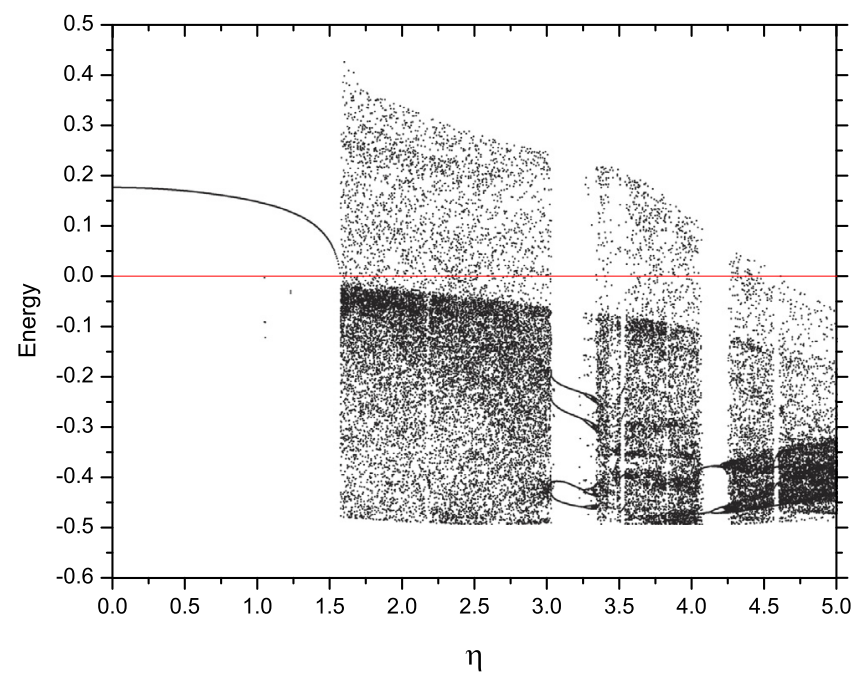

Fig. 9. Diagram of bifurcation for $H_{0}$ as a function of $\eta$, for $\gamma=0$.3. There are ranges of $\eta$ with chaotic attractors, windows of regularity and a period-1 point attractor. The red line is to guide the eyes. (For interpretation of the references to color in this figure legend, the reader is referred to the web version of this article.)

the molecule experiences different scenarios: in the windows of regularity the inter-nuclear distance can possess some discrete values, while in the chaotic attractors, the inter-nuclear distance assumes values in the range $[-0.5,2.5]$. In the lower panel of Fig. 10, we also present the Lyapunov exponent, which exhibits the different regimes of the dynamics.

In Fig. 11 the energy of the system is shown as a function of the dipole amplitude $\varepsilon_{0}$. Up to $\varepsilon_{0} \sim 1.2$, the system remains with negative energy and we can note periodic and chaotic attractors. For $\varepsilon_{0} \in \sim[1.2,1.9]$ the energy can reach positive values; however the particle is trapped in a chaotic attractor. From $\varepsilon_{0} \sim 1.9$ up to $\sim 3.4$, the system is driven to periodic attractors, with positive energy from $\varepsilon_{0} \sim 2$.1. From $\varepsilon_{0} \sim 3.4$ up to 4.0 , the molecule can have positive energies and negative as well. Comparing Fig. 9 with Fig. 11, it can be noticed that the effect of varying $\varepsilon_{0}$ results in attractors with higher energies than the corresponding effect of varying $\eta$. In Fig. 11 the energy reaches the value $\sim 1.7$, while in Fig. 9 the energy is smaller than 0.5 .

Fig. 12 presents the variation of the inter-nuclear distance as function of the laser amplitude $\varepsilon_{0}$, which should be compared with Fig. 10. There is a complex dynamics with periodic and chaotic attractors and the effect of varying $\varepsilon_{0}$ results in attractors with larger inter-nuclear distance in comparison with the effect of varying $\eta$, since in Fig. 12 the value of $x$ reaches almost 6.0, while in Fig. 10 it is smaller than 3.5. In spite of this complexity of the dynamics, we can observe in Figs. 5, 6, 8, 9-12 routes to chaos via flip bifurcations as the corresponding parameter is varied. The Lyapunov exponent reflects all aspects of the dynamics. 

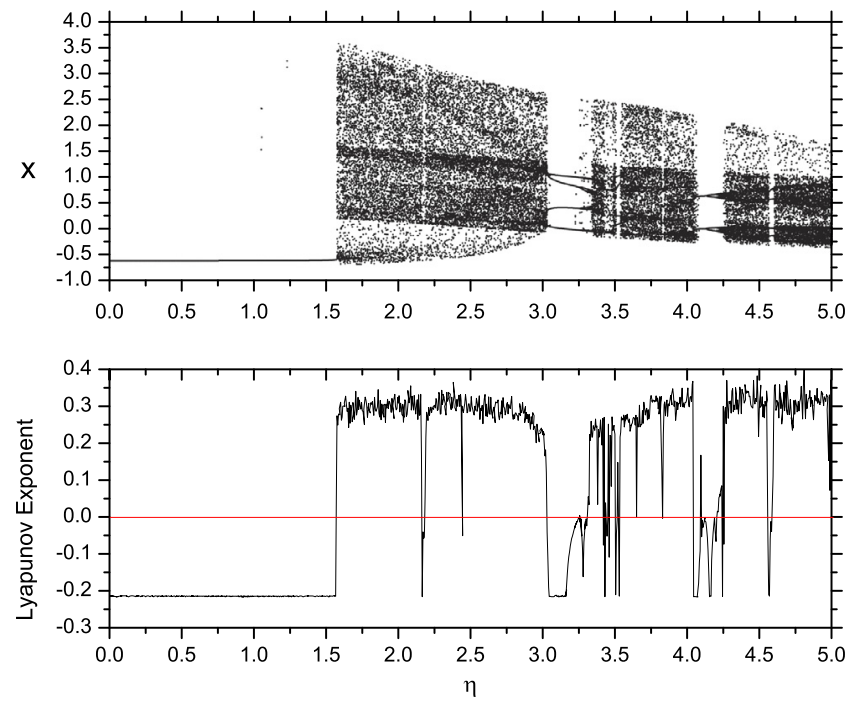

Fig. 10. Upper panel: diagram of bifurcation for the inter-nuclear distance as function of $\eta$, for $\gamma=0.3$. There are values of $\eta$ with chaotic attractors, windows of regularity and a period-1 point attractor, in total agreement with Fig. 9. Lower panel: the corresponding Lyapunov exponent. The red line is to guide the eyes. (For interpretation of the references to color in this figure legend, the reader is referred to the web version of this article.)

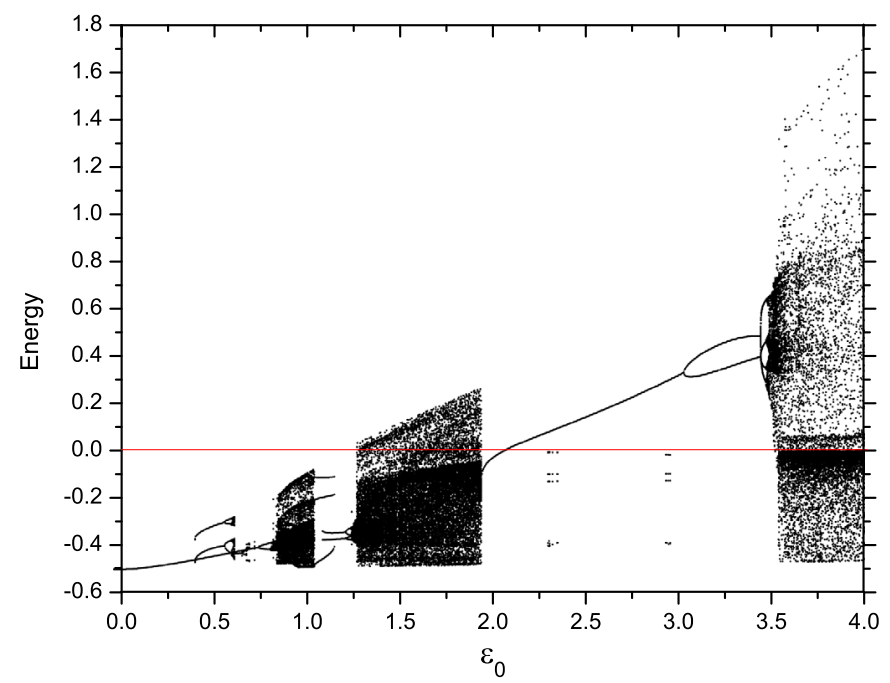

Fig. 11. Diagram of bifurcation for the energy as function of $\varepsilon_{0}$, for $\gamma=0.3$. There are also ranges of $\varepsilon_{0}$ with chaotic attractors, windows of regularity and a period- 1 point attractor. The red line is to guide the eyes. (For interpretation of the references to color in this figure legend, the reader is referred to the web version of this article.)

\section{Conclusion}

In the present work, we have considered the problem of photo-dissociation of polar diatomic molecules modeled by the Morse potential under the effect of time-dependent and position-dependent external forcing.

In the non-dissipative scenario, we have shown that the photo-dissociation can be controlled by generating a non-linear resonance structure with positive energy, that is, the molecule can be trapped in resonant island with positive energy. However, the efficiency of this control mechanism depends on: (i) the initial conditions, which should be inside to an island of the associated resonance structure; and on (ii) the intensity and the non-linearity of the molecule-field interaction.

In the dissipative scenario, the richness of the phase space increases considerably due to the multi-stability introduced in the phase space by chaotic and periodic attractors. Depending on the intensity of the parameters, the nuclei can experience chaotic oscillations, but the molecule may remain bound. The fact that the molecule does not dissociate, even with energy above the dissociation threshold, means that the molecule is trapped in a pseudo bound states, i.e., a high energy state that is sustained by the field interaction and the dissipation. 

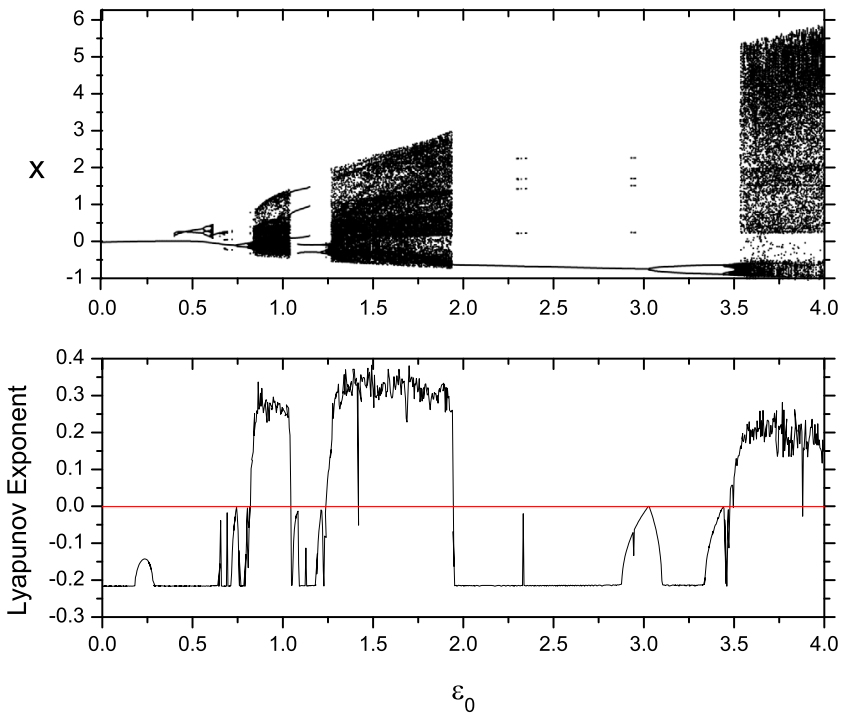

Fig. 12. Upper panel: diagram of bifurcation for the inter-nuclear separation as function of $\varepsilon_{0}$, for $\gamma=0.3$. There are ranges of $\varepsilon_{0}$ with chaotic attractors, windows of regularity and a period-1 point attractor, in total agreement with Fig. 11. Lower panel: the corresponding Lyapunov exponent. The red line is to guide the eyes. (For interpretation of the references to color in this figure legend, the reader is referred to the web version of this article.)

Although it might be expected that dissipation would inhibit dissociation, it is not obvious that this inhibition could occur through chaotic attractors or periodic attractors out of the minimum of the potential well and above the dissociation threshold.

It is worth remarking the global role of the dissipation. With the onset of a small dissipation, all trajectories are driven toward some attractor and the photo-dissociation is completely prevented. As we have shown, the introduction of dissipation, after some transient time, the molecule will be found trapped in an attractor. In summary, our results show that, dissipation prevents the breaking of the chemical bond and by a suitable design of the parameters of the system, the molecule can be trapped in a highly energetic state.

As a final remark, we would like to pose that we have not still investigated the occurrence of crises and their effects on the dynamics, but certainly they could exist. It seems us an interesting point to investigate in a further work, if any destruction of the chaotic attractor via a crisis would favor the dissociation probability.

\section{Acknowledgments}

We acknowledge support from the Brazilian scientific agencies FAPESP-São Paulo Research Foundation through the grants 10/20276-2, 14/00334-9 and 14/23648-9; CNPQ-National Counsel of Technological and Scientific Development grant 473283/2013-1; and CAPES-Coordination for the Improvement of Higher Education Personnel.

\section{References}

[1] R. Kapral, M. Schell, S. Fraser, J. Phys, Chem. 86 (1982) 2205.

[2] C. Scheffczyk, U. Parlitz, T. Kurz, W. Knop, W. Lauterborn, Phys. Rev. A 43 (1990) 6495.

[3] M. Lakshmanan, K. Murari, Chaos in nonlinear oscillations, in: Word Scientific Series on Nonlinear Science, Series A, Vol. 13 , Word Scientific, 1996.

[4] Y. Gu, J.-M. Yuan, Phys. Rev. A 36 (1987) 3788.

[5] M.E. Goggin, P.W. Milonni, Phys. Rev. A 37 (1988) 796

[6] G.R. Graham, M. Hönnerbach, Phys. Rev. A 45 (1992) 5078

[7] V. Constantoudis, C.A. Nicolaides, Phys. Rev. E 64 (2001) 05611.

[8] E.F. de Lima, M.A.M. de Aguiar, Phys. Rev. A 77 (2008) 033406.

[9] A.E. Motter, Y.C. Lai, Phys. Rev. E 65 (2001) 015205

[10] J.M. Seoane, J. Aguirre, M.A.F. Sanjuán, Y.C. Lai, Chaos 16 (2006) 023101.

[11] J.M. Seoane, M.A.F. Sanjuán, Y.C. Lai, Phys. Rev. E 76 (2007) 016208.

[12] E. Ott, T. Tel, Chaos 3 (1993) 417.

[13] P. Gaspard, Chaos, Scattering and Statistical Mechanics, in: Nonlinear Science Series, vol. 9, Cambridge, 2005.

[14] Y.C. Lai, T. Tél, Transient Chaos: Complex Dynamics on Finite Time Scales, in: Applied Mathematical Sciences, vol. 173, Springer, 2001.

[15] J.M. Seoane, M.A.F. Sanjuán, Rep. Progr. Phys. 76 (2013) 016001.

[16] R.B. Walker, R.K. Preston, J. Chem. Phys. 67 (1977) 2017.

[17] J. Stine, D. Noid, Opt. Commun. 31 (1979) 161.

[18] A. Sethy, S. Keshavamutrthy, Phys. Rev. A 79 (2009) 033416.

[19] G.C. Lie, J.-M. Yuan, J. Chem. Phys. 84 (1986) 5486.

[20] W. Knop, W. Lauterborn, J. Chem. Phys. 93 (1990) 3950.

[21] S. Parthasarathy, M. Lakshmanan, Phys. Lett. A 157 (1991) 365.

[22] Z. Jing, J. Deng, J. Yang, Chaos Solitons Fractals 35 (2008) 486. 
[23] S. Behnia, A. Akhshani, M. Panahi, R. Asadi, Acta Phys. Polon. A 123 (2013) 7.

[24] K. Abirami, S. Rajasekar, M.A.F. Sanjuan, Pramana J. Phys. 81 (2013) 127.

[25] G. Gopakumar, M. Abe, M. Kajita, M. Hada, Phys. Rev. A 84 (2011) 062514.

[26] R. Guérout, M. Aymar, O. Dulieu, Phys. Rev. A 82 (2010) 042508.

[27] M.A. Buldakov, V.N. Cherepanov, E.V. Koryukina, Yu.N. Kalugina, J. Phys. B 42 (2009) 105102.

[28] A. Donovan, V. Beltrani, H.J. Rabitz, Phys. Chem. Chem. Phys. 13 (2011) 7348.

[29] V. Beltrani, H.J. Rabitz, Chem. Phys. 137 (2012) 094109.

[30] E.F. de Lima, R. Egydio de Carvalho, Physica D 241 (2012) 1753.

[31] E.F. de Lima, T.N. Ramos, R. Egydio de Carvalho, Phys. Rev. E 87 (2013) 014901.

[32] E.F. de Lima, E.C. Rosado, L.K. Castelano, R. Egydio de Carvalho, Phys. Lett. A 378 (2014) 2657.

[33] A. Wolf, J.B. Swift, H.L. Swinney, J.A. Vastano, Physica D 16 (1985) 285. 